\title{
Effects of synbiotics on intestinal and immune function
}

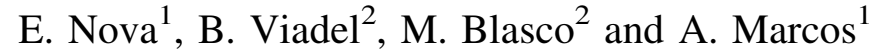 \\ ${ }^{1}$ Immunonutrition Group, Metabolism and Nutrition Department, Instituto del Frío (CSIC), Madrid, Spain and \\ ${ }^{2}$ Department of New Products, AINIA,Technological Center, Valencia, Spain
}

\begin{abstract}
A synbiotic results from the combination of a probiotic and a prebiotic in a single product that is used as a healthy dietary supplement in the restoration and maintenance of colonic flora. The prebiotics most commonly used in the EU are fructo-oligosaccharides (FOS), inulin and galacto-oligosaccharides (GOS). The prebiotic component (non-digestible carbohydrates) selectively increases the survival rate of a particular probiotic or several probiotic strains during intestinal transit and thus their effect on the gastrointestinal tract. In this sense, FOS has been reported to increase bile resistance in bifidobacteria. However, except for the prebiotic effect, the evidence to support the purported effects of synbiotics on health is still scarce in man. There is also the need to establish what differences there are in the observed effects of the synbiotic product $v$. the prebiotic alone. New prebiotics, other than FOS, GOS and inulin, are being assayed for novel applications. $\beta$-Glucans, for example, have shown a lactobacillogenic effect ${ }^{(1)}$ and biotechnology is being employed for the production of human-milk oligosaccharides that might facilitate the development of a healthy microbiota in non-breast-fed infants.

The effects of synbiotic therapies on intestinal function of different types of critically-ill patients have been investigated in a few studies $^{(2)}$. Different synbiotic preparations have also proved useful in preliminary studies of the management of patients with short bowel syndrome $^{(3)}$, ulcerative colitis ${ }^{(4,5)}$, acute pancreatitis ${ }^{(6)}$ and allergy ${ }^{(7)}$. A study has been carried out to evaluate the effects of the consumption for 6 weeks of a synbiotic product containing Lactobacillus acidophilus, Lactobacillus delbrueckii ssp. bulgaricus, Lactobacillus paracasei ssp. paracasei, Streptococcus thermophilus, Bifidobacterium sp. $\left(24 \times 10^{8}\right.$ colony-forming units/d) and FOS on the intestinal microbiota and self-reported intestinal function, as well as on immune function of generally healthy adults. Although no differences in self-reported improvement were found with treatment of mild gastrointestinal symptoms present at baseline such as constipation, flatulence, postprandial bloating or dyspepsia, there was a significant improvement overall in symptoms and in motility in the synbiotic group compared with the placebo group. Intestinal microbiota did not change as a result of synbiotic consumption, and the only significant effect of treatment (ANOVA; $P=0.05$ ) on immune variables was on the concentration of serum soluble L-selectin, which showed a decrease in the synbiotic group. This outcome might lead to a more beneficial profile of the inflammatory markers in relation to the prevention of atherosclerosis and CVD. A trend towards a decrease in CD3-(CD56+16)+ cells (natural killer cells) was found in the women in the synbiotic group. This effect was not observed in men, probably because their values at baseline were at the lower limit of the normal range. No effect of treatment was found in other lymphocyte subsets or other immune variables such as the phagocytic activity of monocytes and granulocytes and inflammatory proteins such as C-reactive protein and caeruloplasmin.
\end{abstract}

1. Snart J, Bibiloni R, Grayson T et al. (2006) Appl Environ Microbiol 72, 1925-1931.

2. Jain PK, McNaught CE, Anderson AD, MacFie J \& Mitchell CJ (2004) Clin Nutr 23, 467-475.

3. Kanamori Y, Sugiyama M, Hashizume K, Yuki N, Morotomi M \& Tanaka R (2004) J Pediatr Surg 39, $1686-1692$.

4. Haskey N \& Dahl WJ (2006) Nutr Rev 64, 132-138.

5. Furrie E, Macfarlane S, Kennedy A, Cummings JH, Walsh SV, O’Neil D \& Macfarlane GT (2005) Gut 54, $242-249$.

6. Olah A, Belagyi T, Poto L, Romics L Jr \& Bengmark S (2007) Hepatogastroenterology 54, 590-594.

7. Ogawa T, Hashikawa S, Asai Y, Sakamoto H, Yasuda K \& Makimura Y (2006) FEMS Immunol Med Microbiol 46, $400-409$. 